\title{
Lesions in Medial Preoptic Area and Bed Nucleus of Stria Terminalis: Differential Effects on Copulatory Behavior and Noncontact Erection in Male Rats
}

\author{
Yian-Cheng Liu, John D. Salamone, and Benjamin D. Sachs \\ Department of Psychology and Biobehavioral Science Graduate Degree Program, University of Connecticut, Storrs, \\ Connecticut 06269-1020
}

The goal of these studies was to assess the regulatory roles of the medial preoptic area (MPOA) and the bed nucleus of the stria terminalis (BST) in sexual arousal, inferred from noncontact erection (NCE) evoked in male rats by remote cues from estrous females. NCE and copulatory behavior were recorded before and after quinolinic acid or radiofrequency (RF) lesions were made in the MPOA (Experiments 1-3) or RF lesions were made in the BST (Experiment 4). All males with MPOA lesions, particularly in the rostral region, displayed severe deficits in copulation but little or no decrement in NCE. In contrast, BST lesions caused relatively moderate deficits in copulation, but they severely impaired NCE. Animals with larger BST lesions, including rostral and caudal medial regions, had more deficits in both copulatory behavior and NCE than did males with smaller lesions confined to the rostral medial BST. These results suggest that (1) the MPOA is critical for copulatory behavior but not for NCE, (2) males that stop copulating after MPOA lesions are still sexually aroused by estrous females, and (3) the BST plays an important role in mediating NCE.

Key words: medial preoptic area; bed nucleus of the stria terminalis; noncontact erection; copulation; sexual arousal; male rats
Why do males fail to copulate after incurring lesions in the medial preoptic area (MPOA)? It has been more than 30 years since the discovery that the MPOA is critical to the expression of normal male copulatory behavior in rats (Heimer and Larsson, 1966/1967), and this finding has been extended to every vertebrate species studied to date (for review, see Meisel and Sachs, 1994). Nonetheless, there is still little consensus on the interpretation of this effect, which has been attributed variously to deficits in sensory or motor function, or in sexual motivation, or in making the transition from motivation to performance (Meisel and Sachs, 1994). It is generally agreed that males with MPOA lesions are sexually motivated, because they continue to investigate estrous females, may make abortive mounting attempts, and engage in learned responses that gain access to an estrous female (Slimp et al., 1978; Everitt and Stacey, 1987; Everitt, 1990).

But are males with MPOA lesions sexually aroused? This question has not been directly assessed in previous research. Males can be motivated to have sex without necessarily experiencing arousal or manifesting it autonomically. The most reliable autonomic index of sexual arousal is penile erection, which is also the index with the greatest face validity and interspecies generality (Zuckerman, 1971; Rosen and Beck, 1988); however, males of most species studied for the effects of MPOA lesions were thought not to develop erections before mounting. In the absence of mounting, there has been no independent measure of the male's sexual

Received Jan. 27, 1997; revised April 14, 1997; accepted April 21, 1997.

This work was supported by a University of Connecticut predoctoral fellowship to Y.-C.L., and by National Science Foundation research Grant IBN-9511247 to J.D.S and National Institutes of Health Grant HD08933 and University of Connecticut Research Foundation Grant UCRF-440526 to B.D.S.

Correspondence should be addressed to Benjamin D. Sachs, Department of Psychology, U-20, University of Connecticut, Storrs, CT 06269-1020.

Copyright (C) 1997 Society for Neuroscience $0270-6474 / 97 / 175245-09 \$ 05.00 / 0$ arousal after MPOA lesions. Rhesus monkeys with MPOA lesions continue to display normal levels of masturbation and associated erection (Slimp et al., 1978), but there were no data on erection during copulation tests, and the erections during masturbation may have been reflexively evoked by somatosensory stimulation rather than induced by remote sexual stimuli or by intrinsic processes.

A recent discovery may offer a way to loosen the Gordian knot of trying to assess sexual arousal in males that no longer mount females. Sachs et al. (1994) demonstrated that rats display penile erections in response to remote cues from estrous females. These "noncontact erections" (NCEs) are analogous to human "psychogenic" erections, usually defined as erections initiated by brain centers in response to visual, auditory, chemosensory, or imaginative stimuli (Krane et al., 1989; de Groat and Booth, 1993; Meisel and Sachs, 1994; Sachs, 1995a). We investigated whether male rats that stopped copulating after incurring MPOA lesions would continue to display NCE, and by inference, sexual arousal.

The bed nucleus of the stria terminalis (BST) has major reciprocal connections with both the MPOA and the medial amygdala (MeA) (Scalia and Winans, 1975; Weller and Smith, 1982; Alheid et al., 1995; Canteras et al., 1995). Bilateral lesions of rat MeA eliminate NCE, whereas they cause a lesser impairment of copulatory behavior (Kondo et al., 1997). Male rats with BST lesions show decrements in several measures of copulation but no change in reflexive erection (Emery and Sachs, 1976; Valcourt and Sachs, 1979; Claro et al., 1995). Because of the major impact of lesions in MeA and MPOA on NCE and copulatory behavior, respectively, and because of the reciprocal connections between these structures and the BST, an understanding of the behavioral regulatory role of this neural system required closer study of the separate effects of BST lesions on copulation and NCE. 


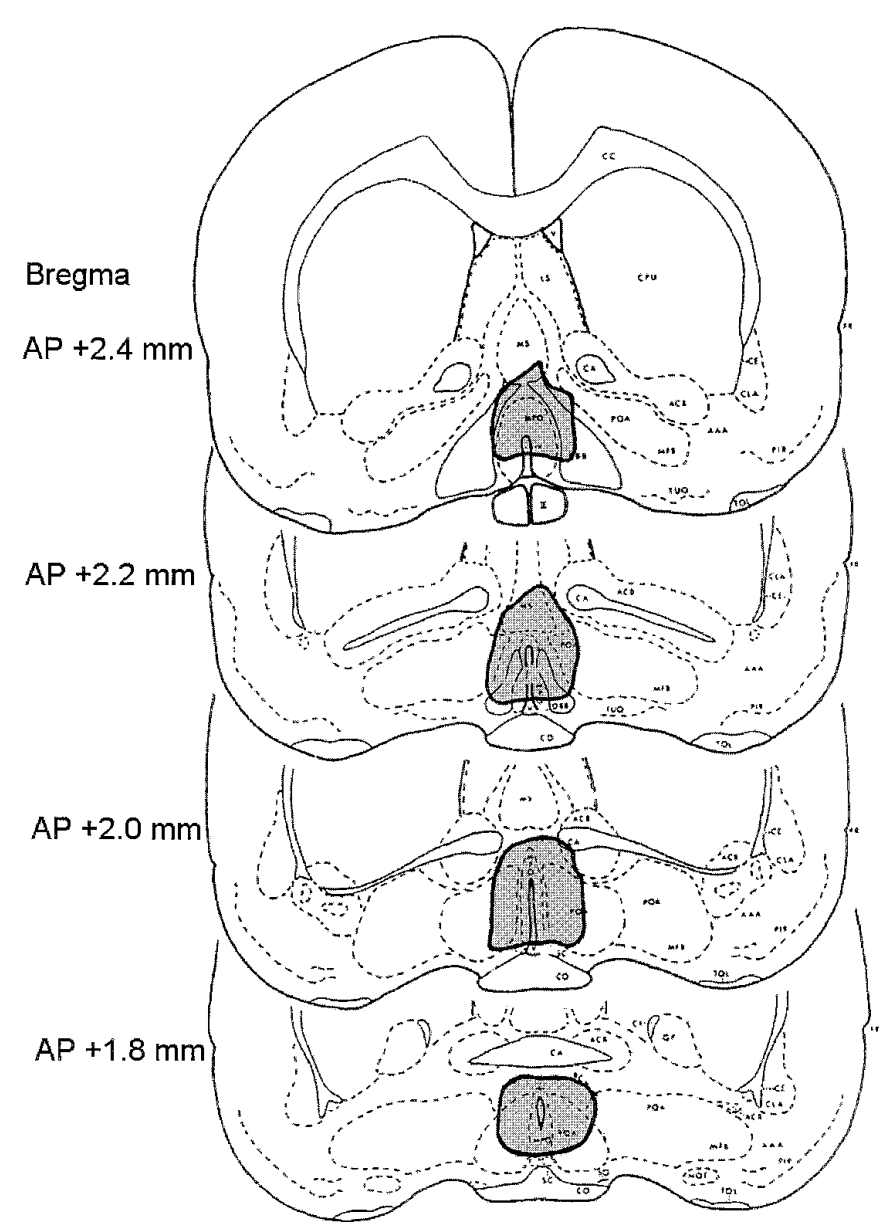

\section{Experiment 1. Median perimeter of MPOA lesions}

Figure 1. Median perimeter of QA lesions in MPOA in Experiment 1, mapped on atlas of Pelligrino et al. (1979).

\section{MATERIALS AND METHODS}

\section{Animals}

Long-Evans rats of Blue Spruce origin (Harlan Sprague Dawley, Indianapolis, IN) were used in these experiments. After arrival in the laboratory, males and females were housed in separate rooms in groups of three to four in wire-mesh cages $(43 \times 34 \times 21 \mathrm{~cm})$, with commercial rodent chow and tap water always available. The colony room temperature was maintained at $23 \pm 1{ }^{\circ} \mathrm{C}$, and lights came on at 11 P.M. and went off at 11 A.M. All behavioral tests were conducted between 1 and 6 P.M., during the dark phase of the light cycle. Female rats were given estradio benzoate $(50 \mu \mathrm{g} / \mathrm{rat}$, s.c.) $48 \mathrm{hr}$ before and progesterone $(500 \mu \mathrm{g} / \mathrm{rat}$, s.c.) $4-6 \mathrm{hr}$ before being used as stimuli in tests of the sexual responses of male rats in all experiments.

Before experimental use, all males were given one to two tests for copulation and NCE as described below. Males were used in these studies only if they copulated to ejaculation and had at least one erection in these screening tests.

\section{Behavioral tests}

Copulation tests. Copulatory behavior was tested in a glass aquarium $(50 \times 27 \times 30 \mathrm{~cm})$ with pine shavings or Sanichips on the floor. A male was given $5 \mathrm{~min}$ to acclimate to the test chamber, after which the test began, defined by introducing a receptive female. The copulation test was terminated after the first ejaculation, or $30 \mathrm{~min}$ after the first intromission if no ejaculation occurred, or $30 \mathrm{~min}$ after introduction of the female if there was no intromission. Occurrences of mounts without intromission, mounts with intromission, and ejaculation were entered into a computer (Holmes et al., 1988) and counted. (Mounts were counted as such only if accompanied by palpation and thrust.) Additional measures derived from

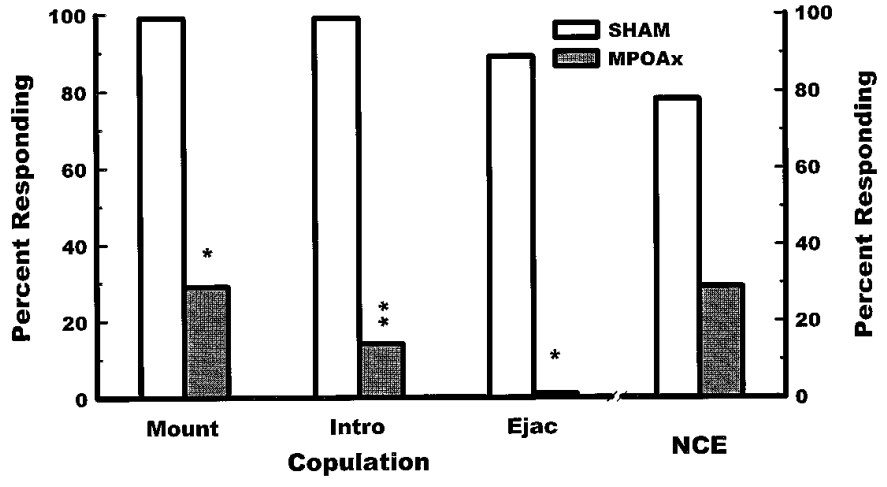

Figure 2. Incidence of mount, intromission, and ejaculation during a copulation test, and of erection in a test for noncontact erection $(N C E)$ in Experiment 1. Males with MPOA lesions $(M P O A x)$ were impaired relative to sham-operated males in the copulation tests, but the difference in incidence of NCE was not reliable. * $p<0.01,{ }^{* *} p<0.001$; Fisher's Exact Probability Test.

event records were latency to first mount, latency to first intromission, ejaculation latency (timed from first intromission), intromission ratio (intromissions divided by the sum of mounts and intromissions; commonly called "hit rate"), and interintromission interval (ejaculation latency divided by number of intromissions).

NCE tests. NCE tests (Sachs et al., 1994) were conducted in a clear Plexiglas chamber $(50 \times 24 \times 30 \mathrm{~cm})$ whose length was divided in half by three partitions placed $1 \mathrm{~cm}$ apart. The center partition had four equally spaced "windows" $(2.5 \times 9 \mathrm{~cm})$; the other partitions had four equally spaced "doors" $(2.5 \times 7.5 \mathrm{~cm})$ aligned below the windows. Thus, the chamber prevented direct contact between the animals but allowed reciprocal auditory, visual, and olfactory communication. Some NCE tests in Experiments 1 and 2 used test chambers of similar size, divided in half by two sheets of wire mesh placed $2.5 \mathrm{~cm}$ apart. Both kinds of test chambers were littered with pine shavings or Sanichips. Male rats were placed individually in one side of the test chamber. After $5 \mathrm{~min}$ acclimation, the test was started by putting a receptive female into the other side. Erections were scored when the penis emerged from the penile sheath, which was accompanied by penile grooming and hip flexion (Sachs et al., 1994). The latency to first erection and the number of erections were recorded. The number of nose pokes by the male through the "doors" was recorded as a measure of the male's attention to the female in Experiments 3 and 4 . The NCE test was terminated $15 \mathrm{~min}$ after the first erection or $20 \mathrm{~min}$ after introducing the female if no erection occurred, except in Experiment 1, in which each male was tested for $25 \mathrm{~min}$ whether or not erection occurred.

\section{Histology}

After completion of all behavioral tests, males were anesthetized deeply and perfused intracardially with $0.9 \%$ saline followed by $10 \%$ formalin. Brains were removed and stored in $10 \%$ formalin. Frozen sections were cut at $50 \mu \mathrm{m}$ in the coronal plane through the target regions and stained with cresyl violet. Because of differences among experiments in the placement of the skull during stereotaxic surgery, the atlas of Pelligrino et al. (1979) was used to analyze and depict the lesions for Experiments 1 and 2, whereas the atlas of Paxinos and Watson (1986) was used for Experiments 3 and 4.

\section{Statistical analysis}

Proportional data were analyzed by comparing lesion groups with shamoperated groups using the Fisher's Exact Probability Test (Statxact for Systat, Evanston, IL). Latency data were evaluated by survival analyses, including Kaplan-Meier statistics (SPSS, Chicago, IL). Other data were analyzed by repeated measures ANOVA using software from SAS or SPSS. For all statistics, significance was inferred when $p<0.05$.

\section{Experimental protocols}

Experiment 1: Quinolinic acid $(Q A)$ lesion of the MPOA. To understand the role of the MPOA in regulating erection, especially the NCE response in male rats, the excitotoxin QA (Sigma, St. Louis, MO) was used to destroy the nerve cell bodies while sparing the nerve fibers to or passing 


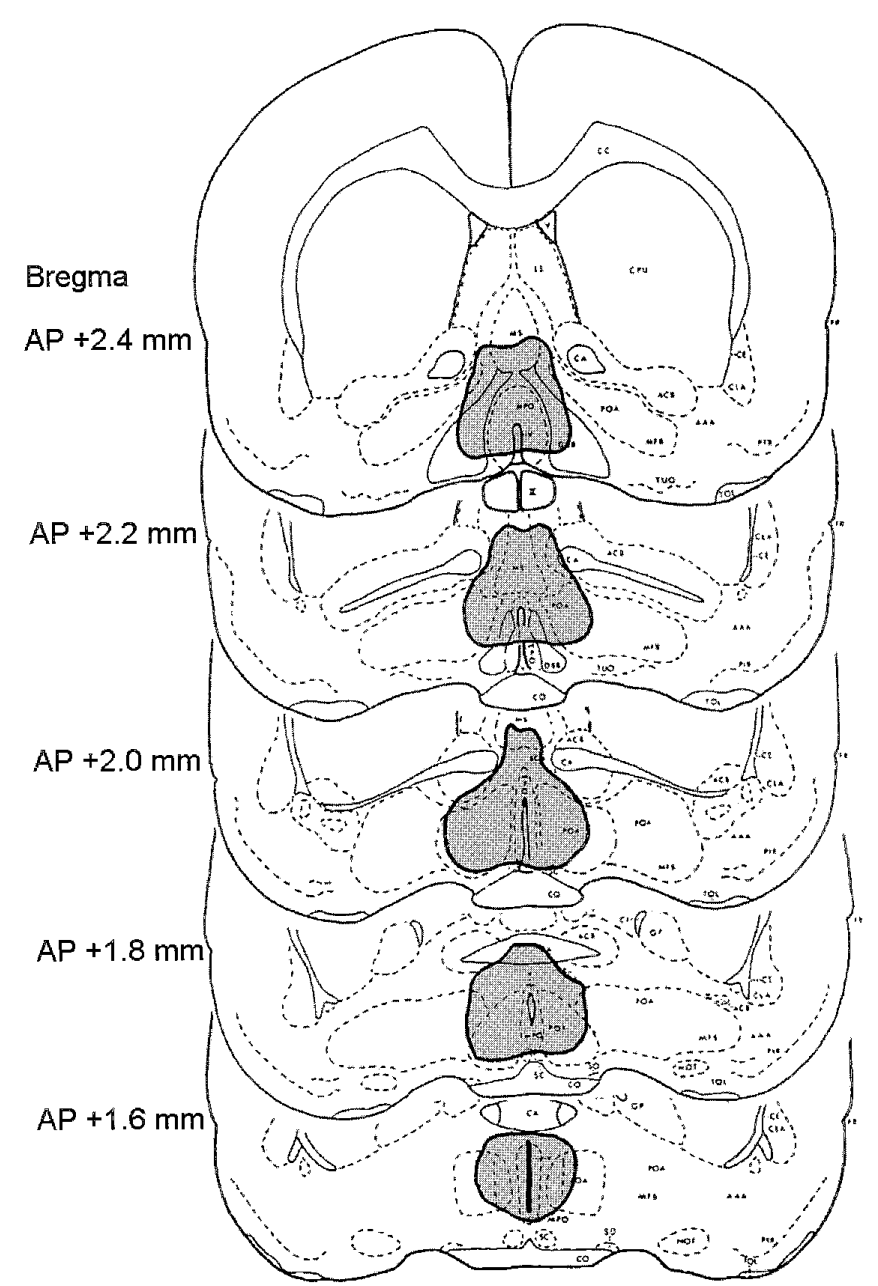

Experiment 2. Median perimeter of MPOA lesions

Figure 3. Median perimeter of QA lesions in MPOA in Experiment 2, mapped on atlas of Pelligrino et al. (1979).

through the target region (Winn, 1991). Male rats $(n=18), 280-300 \mathrm{~d}$ old at the start of the study, were randomly divided into an MPOA-lesion group (MPOAx, $n=9$ ) and a sham-lesion group (Sham, $n=9$ ). For stereotaxic surgery, we anesthetized the males with sodium pentobarbital $(60 \mathrm{mg} / \mathrm{kg}$, i.p.; Butler) and placed them with the incisor bar at $5 \mathrm{~mm}$ above the interaural line. After a midline incision $(1.5-2.0 \mathrm{~cm})$, a burr hole was made on the skull, and four stainless steel screws were placed on each side about the burr hole. A pair of stainless steel 23 ga guide cannulae (16 mm long) were implanted $1.0 \mathrm{~mm}$ above the MPOA region bilaterally. The stereotaxic coordinates for the cannulation of the MPOA region relative to bregma were anterior-posterior $(\mathrm{AP})+2.2 \mathrm{~mm}$, medial-lateral (ML) $\pm 0.5 \mathrm{~mm}$, dorsal-ventral (DV) $-7.3 \mathrm{~mm}$ (from the surface of the skull) (Pellegrino et al., 1979). Dental cement was applied around the cannulae and screws and allowed to dry. Three weeks after the MPOA cannulation, each male, under sodium pentobarbital anesthesia $(60 \mathrm{mg} / \mathrm{kg})$, was injected with either QA, $120 \mathrm{nmol}$ in $0.4 \mu \mathrm{l}$ per side, or

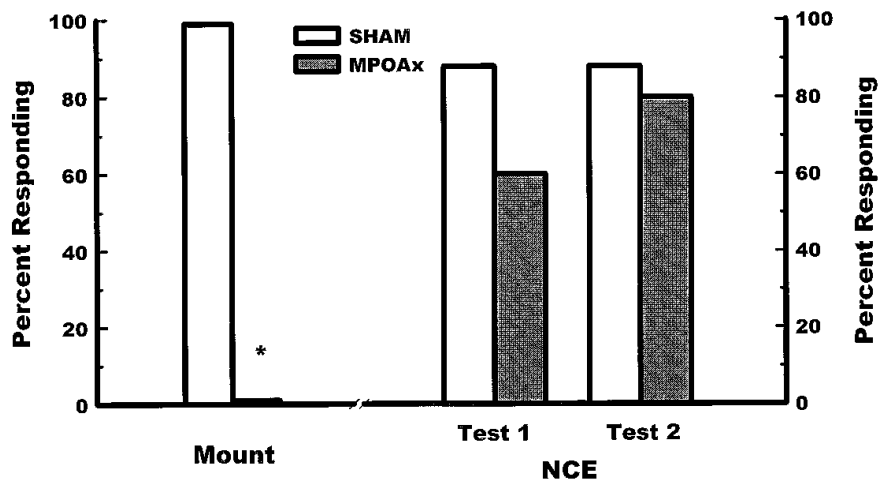

Figure 4. Incidence of mount during a copulation test, and of erection in two tests for noncontact erection (NCE), in Experiment 2. No males with MPOA lesions (MPOAx) mounted, but the difference in incidence of NCE was not reliable in either test. * $p<0.001$; Fisher's Exact Probability Test.

vehicle, $0.4 \mu \mathrm{l}$ per side, using two $10 \mu \mathrm{l}$ Hamilton syringes linked with a pair of 30 ga stainless steel injection tips $(17 \mathrm{~mm}$ long) through the implanted cannulae. The Hamilton syringes were operated by a Harvard 22 microprocessor syringe pump (Harvard Apparatus, South Natick, MA) with the injection speed at $0.2 \mu \mathrm{l} / \mathrm{min}$. The injection tips remained in place for $5 \mathrm{~min}$ after injection before withdrawal; then the implanted cannulae were sealed by dental cement. Four to five days after QA or vehicle injection, each male was tested for NCE and for copulation in counterbalanced order. The interval between the two tests was $4 \mathrm{~d}$.

Experiment 2: $Q A$ lesion of the MPOA. This study was a partial replication and extension of Experiment 1, with the intention of reducing damage to tissue outside of the MPOA by using a lower dose, higher concentration, and slower injection speed of QA. In addition, infusions were performed at the time of surgery, rather than via insertion of permanent cannulae.

Male rats, $100-110 \mathrm{~d}$ old, were randomly assigned to the lesion group (MPOAx, $n=13$ ) or the sham-operation group (Sham, $n=8$ ). After anesthesia (sodium pentobarbital, $60 \mathrm{mg} / \mathrm{kg}$, i.p.) each male was placed in a stereotaxic apparatus as in Experiment 1. After a burr hole was made in the skull, a pair of 30 ga stainless steel guide cannulae, which linked up with two $10 \mu \mathrm{l}$ Hamilton syringes, were lowered directly to the MPOA regions on both sides. The stereotaxic coordinates for the MPOA relative to bregma were AP $+2.4 \mathrm{~mm}, \mathrm{ML} \pm 0.5 \mathrm{~mm}, \mathrm{DV}-8.8$ $\mathrm{mm}$ (from the surface of the skull) (Pellegrino et al., 1979). QA (105 $\mathrm{nmol}$ in $0.28 \mu \mathrm{l}$ per side) or the same volume of the vehicle for sham operation was injected bilaterally by a Harvard 22 microprocessor syringe pump with an injection speed of $0.07 \mu \mathrm{l} / \mathrm{min}$. The cannula tips remained in place for 6-8 min after injection before withdrawal, and then the incision was closed with $11 \mathrm{~mm}$ wound clips. After 6-7 d recovery all males were given an NCE test (NCE Test 1), followed 3-4 $\mathrm{d}$ later by a copulation test (Copulation Test). After another 4-5 d, all males were retested for NCE (NCE Test 2).

Experiment 3: Radiofrequency (RF) lesion of the MPOA. In this study we used RF lesion through an RFG-4A lesion maker and TCZ electrodes (Radionics Inc., Burlington, MA), which destroyed both neurons and axons passing through the lesions sites. Each male was placed on a stereotaxic apparatus after anesthesia, and the incisor bar was set at 3.3 $\mathrm{mm}$ below the interaural line. The stereotaxic coordinates for the MPOA lesions relative to bregma were AP $+0.2 \mathrm{~mm}, \mathrm{ML} \pm 0.5 \mathrm{~mm}, \mathrm{DV}-8.6$ $\mathrm{mm}$ (or DV $-7.6 \mathrm{~mm}$ for sham surgeries) from the surface of the skull (Paxinos and Watson, 1986). For each male in the MPOA lesion group

Table 1. Latency and number of noncontact erections (NCEs) in Experiment 2

\begin{tabular}{lccccc} 
& \multicolumn{2}{c}{ Test 1} & & Test 2 & \\
\cline { 2 - 3 } \cline { 5 - 6 } & MPOAx & Sham & & MPOAx & Sham \\
\hline NCE latency (min) & $2.59 \pm 1.19$ & $4.80 \pm 0.97$ & & $3.53 \pm 1.25$ & $5.91 \pm 1.68$ \\
No. of NCEs & $4.7 \pm 0.9$ & $3.7 \pm 0.4$ & & $4.5 \pm 1.3$ & $3.6 \pm 0.5$
\end{tabular}

Rats received QA-induced lesions in MPOA (MPOAx; $n=5$ ) or sham lesions (Sham; $n=8)$. Values are mean \pm SEM. None of the differences between groups or tests was reliable. 


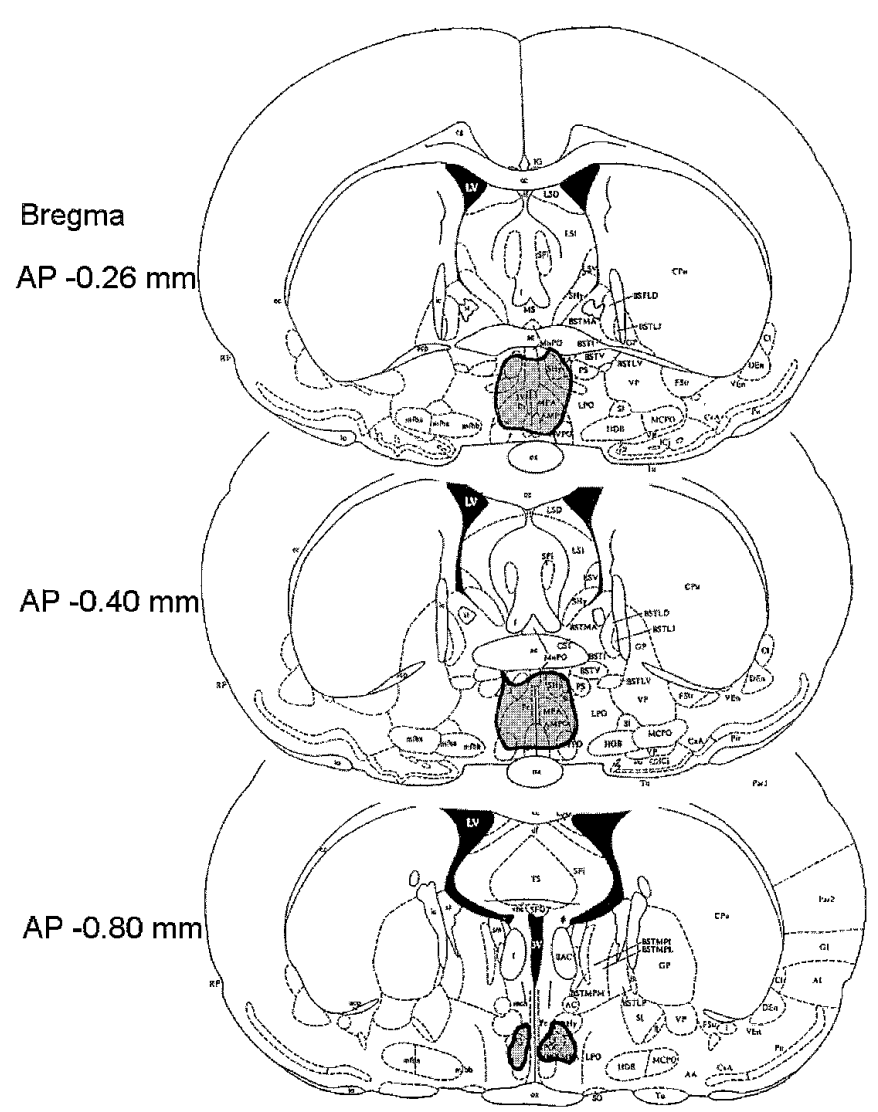

\section{Experiment 3. Median perimeter of MPOA lesions}

Figure 5. Median perimeter of RF lesions in MPOA in Experiment 3, mapped on atlas of Paxinos and Watson (1986).

(MPOAx, $n=18$ ), the electrode (tip diameter $0.25 \mathrm{~mm}$, tip exposure 0.25 $\mathrm{mm}$ ) was lowered to the target region through a burr hole on one side and then the other side. Tip temperature was set at $83^{\circ} \mathrm{C}$ for $75 \mathrm{sec}$. For males with sham surgeries (Sham, $n=10$ ), the electrode was lowered to $1.0 \mathrm{~mm}$ above the MPOA regions, and no heat was generated. The incision was closed with $11 \mathrm{~mm}$ wound clips. Nine to eleven days after surgery, males were given an NCE test (NCE Test 1), followed $1 \mathrm{~d}$ later by a copulatory test (Copulation Test 1). Four days after Copulation Test 1, each male was retested for NCE (NCE Test 2), and another copulation test (Copulation Test 2) ensued 1-2 d later.

Experiment 4: RF lesion of the BST. The males were 80-90 d old at the start of this study. Twenty males received bilateral BST lesions, and 10 males had sham operations. The surgical procedure was similar to Experiment 3, except that the RF lesion temperature was set at $76^{\circ} \mathrm{C}$ for 60 sec. The stereotaxic coordinates for the BST regions relative to bregma were AP $-0.2 \mathrm{~mm}, \mathrm{ML} \pm 1.5 \mathrm{~mm}, \mathrm{DV}-6.5 \mathrm{~mm}$ from the surface of the brain (or DV $-5.0 \mathrm{~mm}$ for sham operation) (Paxinos and Watson, 1986). Each male was tested for NCE (NCE Test 1) 9-10 d after surgery,

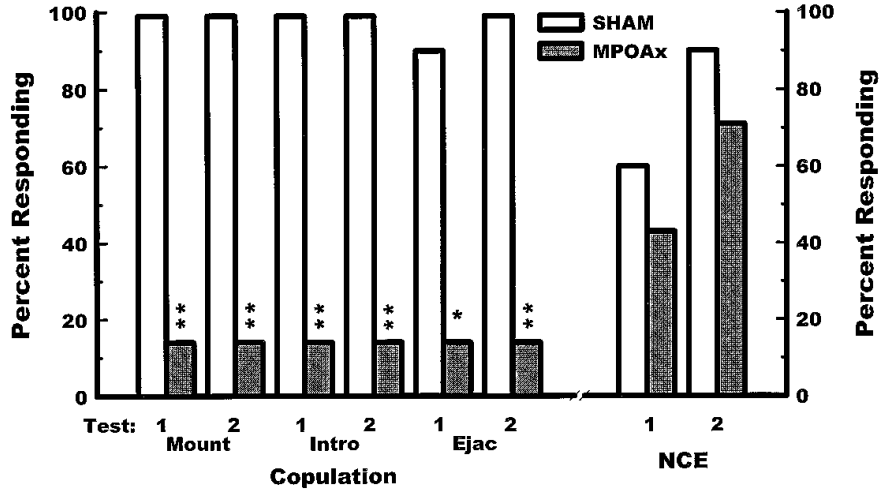

Figure 6. Incidence of mount, intromission, and ejaculation during a copulation test, and of erection in two tests for noncontact erection $(N C E)$, in Experiment 3. Males with MPOA lesions (MPOAx) were impaired on all copulatory measures, but the difference in incidence of NCE was not reliable in either test. ${ }^{*} p<0.01,{ }^{* *} p<0.001$; Fisher's Exact Probability Test.

followed by a copulatory test (Copulation Test) $1-2 \mathrm{~d}$ later, followed after $4-5 \mathrm{~d}$ by another NCE test (NCE Test 2).

\section{RESULTS}

\section{Experiment 1: QA lesion of the MPOA}

Histology revealed well placed bilateral lesions in seven MPOAx males (Fig. 1), with approximately two thirds of the entire rostrocaudal extent of the MPOA being destroyed. In some cases lesions also invaded the diagonal band of Broca, lateral preoptic area, and medial septal nucleus.

In the postoperative copulatory test (Fig. 2), all the MPOAx males continued to investigate the estrous female and to make abortive mount attempts, but only two of seven MPOAx males mounted, versus nine of nine Sham males $(p<0.01)$; only one of seven MPOAx males intromitted, versus nine of nine Sham males $(p<0.001)$; and no MPOAx male ejaculated, compared with eight of nine Sham males $(p<0.01)$. In the NCE test (Fig. 2), two of seven MPOAx males displayed NCEs, whereas seven of nine Sham males displayed NCEs (NS). The latencies to first NCE did not differ reliably between the groups $(\log \operatorname{Rank}=2.81$; $\mathrm{df}=1$; $p=0.09)$.

\section{Experiment 2: QA lesion of the MPOA}

The change from Experiment 1 in the method of excitotoxin infusion did not yield smaller or more reliable lesions. Bilateral lesions extending from rostral to caudal ends of the MPOA were present in five MPOAx males (Fig. 3). Some lesions also extended into part of the diagonal band of Broca, lateral preoptic area, medial septal nucleus, and anterior commissure (Fig. 3).

In the copulatory test after the surgeries, as in Experiment 1,

Table 2. Latency and number of noncontact erections (NCEs) and number of nose pokes through partitions in Experiment 3

\begin{tabular}{lccccc} 
& \multicolumn{2}{l}{ Test 1} & & Test 2 & \\
\cline { 2 - 3 } \cline { 5 - 6 } & MPOAx & Sham & & MPOAx & Sham \\
\hline NCE latency (min) & $10.81 \pm 5.43$ & $8.64 \pm 2.12$ & & $4.79 \pm 1.63$ & $4.00 \pm 1.06$ \\
No. of NCEs & $1.3 \pm 0.3$ & $3.7 \pm 0.8$ & & $1.8 \pm 0.2$ & $2.8 \pm 0.4$ \\
No. of nose pokes & $55.9 \pm 15.4$ & $67.4 \pm 9.7$ & & $45.9 \pm 5.9$ & $71.4 \pm 11.7$
\end{tabular}

Rats received RF-induced lesions in MPOA (MPOAx; $n=7$ ) or sham lesions (Sham; $n=10$ ). Values are mean \pm SEM. None of the differences between groups or tests was reliable. 


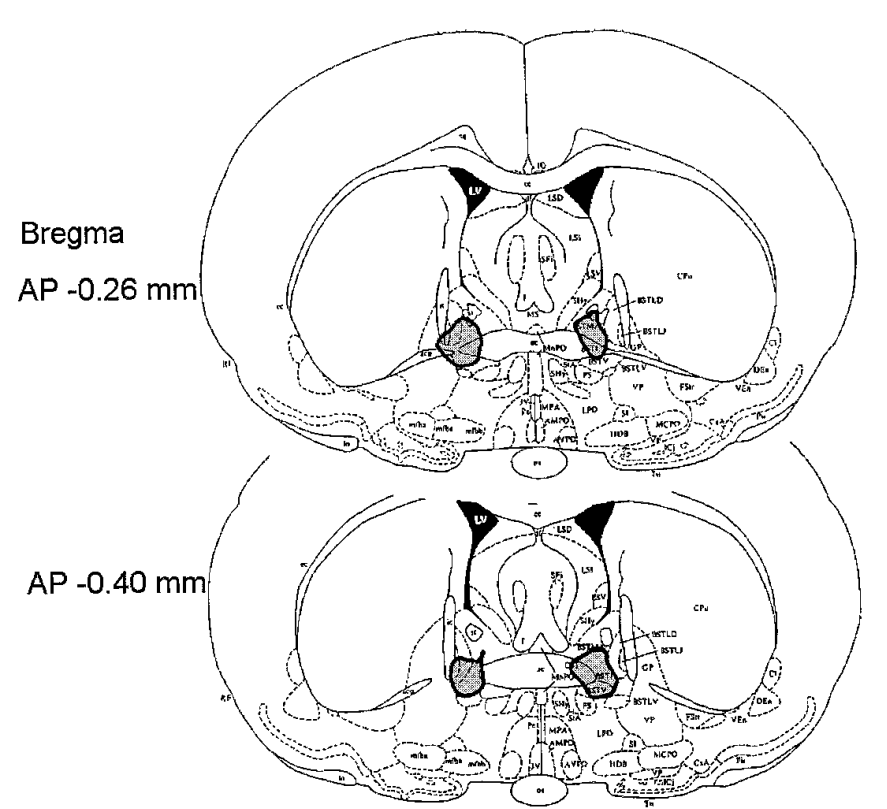

BSTx-S lesions

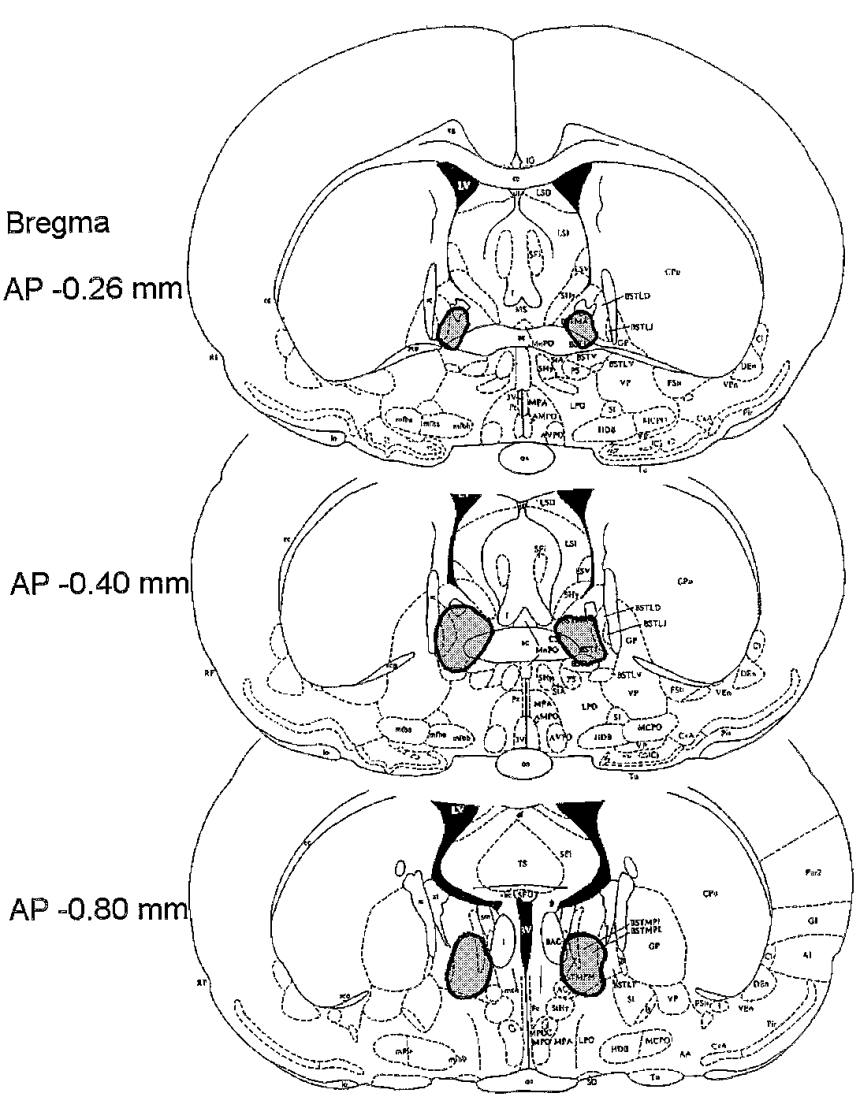

BSTx-L lesions

Figure 7. Median perimeter of RF lesions in BST in Experiment 4, mapped on atlas of Paxinos and Watson (1986). Males were divided into two groups according to the size of their lesions. Left, Small lesions $(B S T x-S)$ extending posterior a little or not at all beyond bregma $-0.40 \mathrm{~mm}$. Right, Large lesions $(B S T x-L)$, extending posterior at least as far as bregma $-0.80 \mathrm{~mm}$.

the MPOAx males investigated the estrous females, but none of the five MPOAx males mounted, whereas all eight Sham males ejaculated ( $p<0.001)$ (Fig. 4). In NCE Tests 1 and 2 (Fig. 4), however, there was not a significant difference between MPOAx and Sham males in the proportion of rats displaying NCE. Of MPOAx males, three of five (Test 1) and four of five (Test 2) displayed NCE, compared with seven of eight Sham males in each test. There was also no significant difference between groups in either test in the number of erections or the latency to the first NCE (Table 1).

\section{Experiment 3: RF lesion of the MPOA}

Seven males had bilateral lesions in the MPOA, primarily in the rostral portion (usually including the periventricular hypothalamic nucleus and anterior medial preoptic nucleus), with some sparing of the caudal MPOA. In some cases the lesions extended into part of the lateral preoptic area, septohypothalamic nucleus, or central medial preoptic nucleus (Fig. 5).

Only one of the seven MPOAx males achieved mount, intromission, and ejaculation in both copulation tests. Among Sham males, in contrast, all 10 rats mounted and intromitted in both tests $(p<0.001), 9$ of $10(p<0.01)$ ejaculated in the first test, and 10 of $10(p<0.001)$ ejaculated in the second test (Fig. 6). NCEs were displayed by three of seven and five of seven MPOAx males in NCE Tests 1 and 2, respectively; the comparable rates for Sham males were 6 of 10 (Test 1, NS) and 9 of 10 (Test 2, NS) (Fig. 6). In addition, there were no significant differences between the MPOAx and Sham males in the number of nose pokes or NCEs, or in the latencies to the first NCE in either test (Table 2).

During Copulation Test 1, we observed that some MPOAx males exhibited behavior similar to NCE, including penile erections and associated activity. Therefore, we recorded this behavior more systematically in Copulation Test 2. Among the six MPOAx males that failed to mount, four males displayed NCE-like patterns. For these rats, the mean latency to the first erection was longer than their NCE latency in NCE Test 2 (16.6 \pm 3.9 vs $5.7 \pm$ $1.8 \mathrm{~min} ; t(6)=2.55 ; p=0.043)$.

\section{Experiment 4: RF lesion of the BST}

After histological examination, the 15 males with bilateral BST lesions were divided into two groups. In those rats with large lesions (Group BSTx-L, $n=9$ ), BST lesions extended throughout most of the rostrocaudal extent of the BST, including bregma $-0.8 \mathrm{~mm}$ (Fig. 7). In rats with smaller lesions (Group BSTx-S, $n=$ 6 ), the rostral portion was destroyed but the caudal part was largely spared (Fig. 7). In both groups, some lesions also extended into part of the anterior commissure, dorsal part of the lateral division of the BST, ventral division of the BST, and the internal capsule.

In the postoperative copulatory test (Fig. 8), the incidence of mounting, intromitting, and ejaculating was similar for Sham and BSTx-S males. Relative to Sham males, however, fewer BSTx-L males achieved intromission $(p=0.03)$ and ejaculation $(p<$ $0.01)$. Some measures of copulation also distinguished the groups. 
Table 3. Number of nose pokes during two tests for noncontact erection and measures of copulation in Experiment 4

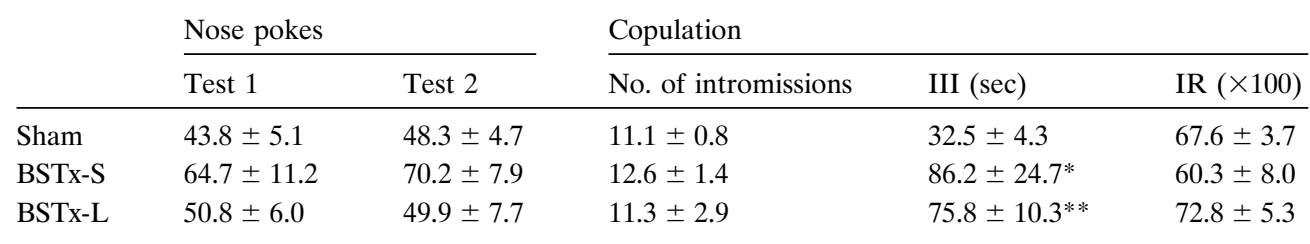

Rats received RF lesions in the bed nucleus of the stria terminalis (BSTx) or sham lesions (Sham; $n=10)$. After histological examination, BSTx males were assigned to those that had small (BSTx-S; $n=6$ ) or large lesions (BSTx-L; $n=9$ ). Values are mean \pm SEM. III, Interintromission interval; IR, intromission ratio. ${ }^{*} p<0.05$ and ${ }^{*} p<0.01$, relative to Sham, by least significant difference post hoc test; for overall ANOVA $F_{(2,15)}=4.95, p<0.02$.

Groups BSTX-S and BSTx-L were similar on all measures, but compared with Sham males, both groups had longer interintromission intervals (Table 3) and longer ejaculation latencies (Fig. $9 A$ ). The groups had similar numbers of intromissions and intromission ratios (Table 3 ).

Lesion-induced impairments were also evident in NCE (Fig. 8). Relative to Sham males, the incidence of NCE in Test 1 was lower for both BSTx-S and BSTx-L males (for both groups, $p<0.01$ by Fisher's Exact Probability Test). In Test 2, only the BSTx-L males had a reliably lower incidence of NCE $(p<0.001)$. In both Tests 1 and 2, the BSTX-L and BSTx-S males had longer NCE latencies than Sham males (Fig. 9B, $C ; p<0.01$ ), but the difference between the two BSTx groups was not reliable. The number of nose pokes was similar among the three groups (Table 3).

\section{DISCUSSION}

Lesions of the MPOA and BST had dramatically different effects on NCE and copulatory behavior. MPOA lesions abolished copulation but had little or no effect on NCE, whereas BST lesions had a much smaller effect on copulation but caused severe deficits in NCE. Clearly, these two sexual responses differ in their neural mediation. We discuss the results of MPOA and BST lesions in turn.

\section{MPOA lesions stopped copulation but not NCE}

Experiments 1-3 confirmed many previous reports that MPOA lesions severely disrupt the initiation of copulatory behavior in

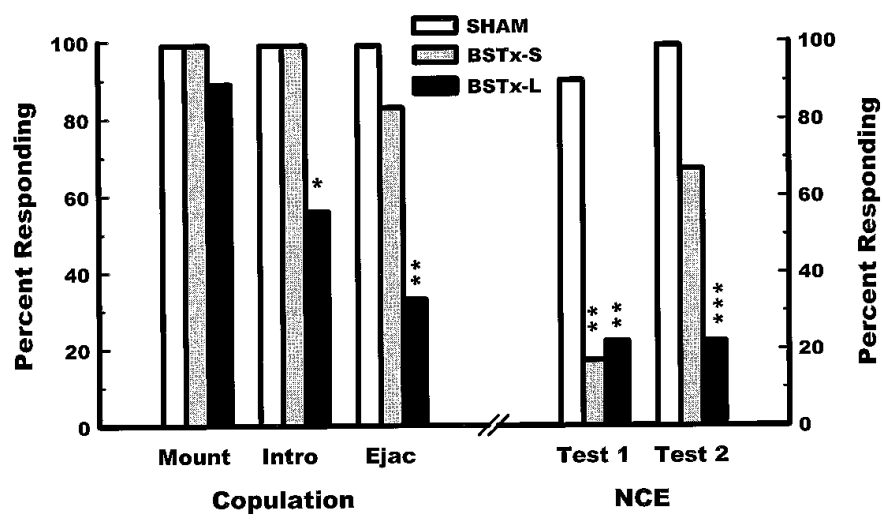

Figure 8. Incidence of mount, intromission, and ejaculation during a copulation test, and of erection in two tests for noncontact erection $(N C E)$, in Experiment 4. Males with large BST lesions $(B S T x-L)$ were impaired on most measures of copulation, but males with small BST lesions $(B S T x-S)$ were similar to sham-lesion males. Relative to Sham males, the reduced incidence of NCE was reliable for both BSTx groups in Test 1 , but only for BSTx-L males in Test $2 .{ }^{*} p<0.05,{ }^{*} * p<0.01$, *** $p<0.001$; Fisher's Exact Probability Test. male rats (for review, see Everitt and Stacey, 1987; Meisel and Sachs, 1994). Excitotoxic lesions in the MPOA (Experiments 1 and 2) were as effective as RF lesions (Experiment 3) in impairing
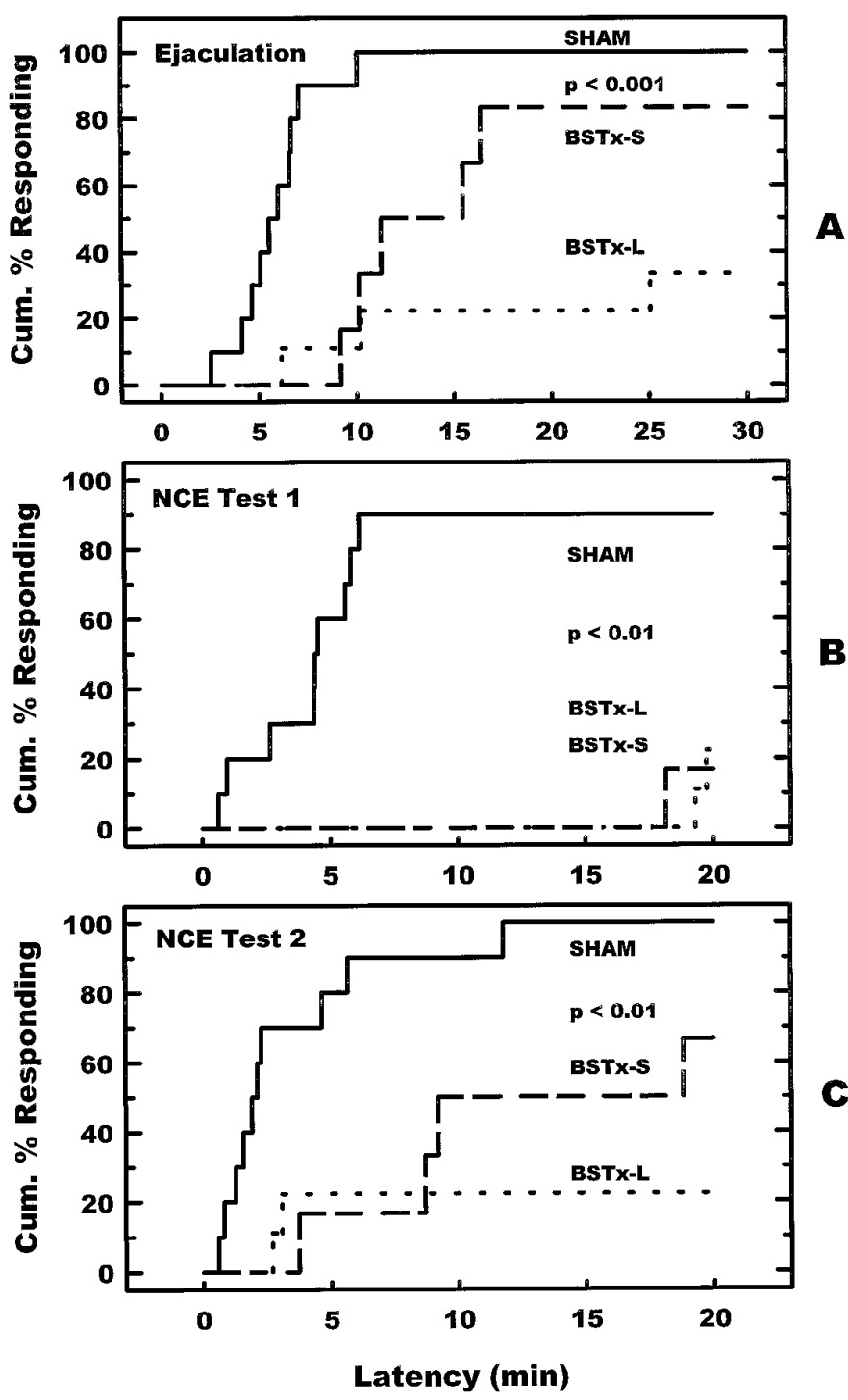

Figure 9. Cumulative percentage of males responding over the course of testing after large lesions $(B S T x-L)$, small lesions $(B S T x-S)$, or sham lesions (Sham) in the BST. Both BSTx groups differed reliably from Sham males, but not from each other, in ejaculation latency during the test for copulation $(A)$ ( $\log$ Rank $=$ 26.91; df =2), and in erection latency during noncontact erection (NCE) test 1 (B) $(\log$ Rank $=21.02 ; \mathrm{df}=2)$ and test $2(C)(\log \operatorname{Rank}=19.44 ; \mathrm{df}=2)$. 
Table 4. Incidence of noncontact erection in Experiments 1-3 after sham or actual lesions in the medial preoptic area (MPOA)

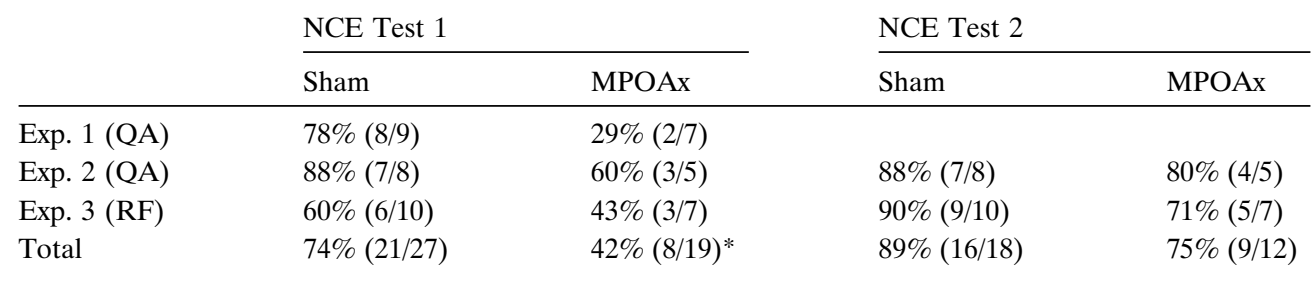

Only when totaled across the three experiments was there a reliable difference in the incidence of NCE, and then only in Test

1. In Experiment 1, the males were tested only once. ${ }^{*} p=0.04$; Fisher's Exact Probability test.

copulation, indicating that neurons intrinsic to the MPOA are essential to this function (Hansen et al., 1982).

No special effort was made to localize the effects within the MPOA-anterior-hypothalamic continuum (MPOA-AH). Still, it may be noteworthy that most of the rostrocaudal extent of the MPOA was destroyed in all three studies, but the most caudal part was largely unaffected in Experiment 3, and the anterior hypothalamic area was spared in all three experiments (Figs. 1, 3, 5). These results may indicate that the integrity of the rostral MPOA is more important for preserving copulatory function. In contrast, some investigators (e.g., Van de Poll and van Dis, 1979) have concluded that the caudal MPOA and anterior hypothalamic portions are the critical ones. Unlike the gerbil, in which lesions limited to a single nucleus within the MPOA eliminate copulation (Yahr and Gregory, 1993), one cannot yet identify a single site in the rat MPOA-AH that is essential for mating.

The MPOA lesions that impaired copulation had, at most, a small and transient effect on NCE. There was not a reliable proportional difference between MPOAx and Sham males displaying NCE in any of the five tests among the three experiments. When data were summed across experiments, the percentage of males exhibiting NCE in Test 1 was reliably lower for MPOAx males than for Sham males (Table 4); however, 5-8 d later in NCE Test 2 (Experiments 2 and 3 only) the groups responded similarly. It is premature to speculate on whether this small apparent recovery of function, if replicable, is attributable to a reduction in nonspecific effects of the lesion or to residual MPOA tissue assuming the functions of damaged tissue.

\section{MPOA role in erectile function}

The role of the MPOA in erectile function and sexual arousal has been ambiguous. Electrical stimulation of rat MPOA can induce penile erections in the absence of estrous females (Malsbury, 1971; Courtois and Macdougall, 1988; Paredes et al., 1990), even in anesthetized males (Giuliano et al., 1996). Pharmacological treatments of the MPOA can also stimulate or inhibit erectile function in and ex copula (Hull, 1995). In all these experiments, however, the MPOA was not isolated from the many brain structures with which it has reciprocal connections. It is uncertain therefore whether the observed effects on erection resulted directly from actions on the MPOA or from indirect effects exerted on other brain structures, rostral and caudal to the MPOA, that may not normally be stimulated by physiological activity in the MPOA.

Correlative evidence has also supported a role for the MPOA in erectile function. Catecholaminergic (mainly dopaminergic) activity increases in the MPOA when male rats are exposed to inaccessible females in an environment similar to our NCE test
(Blackburn et al., 1992; Hull et al., 1995). Also, neurophysiological recording from the MPOA of male rats (Shimura et al., 1994) and monkeys (Oomura et al., 1983) has shown increased activity of some MPOA neurons during precopulatory behavior. These signs of MPOA activity do not necessarily reflect the excitation specifically associated with NCE, because bedding soiled by estrous females is attractive to males and promotes dramatic changes in hormones and in MPOA neurochemistry (Pfaus and Phillips, 1991; Meisel and Sachs, 1994; Hull, 1995; Mas, 1995) but does not evoke NCE (Sachs et al., 1994). Therefore, it remains to be determined what the relation is between MPOA activity in response to females or soiled bedding and those processes that give rise to erection generally and NCE in particular.

Other evidence suggests that the MPOA may not be critical for erection. We have already considered the ambiguous data from rhesus with MPOA lesions (Slimp et al., 1978). Reflexive erections (i.e., in response to somesthetic stimulation) can be evoked in intact, conscious rats by holding them in supine position and tonically retracting the penile sheath (Hart, 1968; Sachs and Garinello, 1978). These erections are not affected by MPOA lesions (Stefanick and Davidson, 1987), but the supine test does not constitute natural sexual stimulation, and the brain's regulation of reflexive erections may be quite different from its regulation of erections in other contexts (Sachs, 1995a,b).

The maintenance of NCE by MPOAx males in the present experiments demonstrates that erections evoked by remote sexual stimuli do not require an intact MPOA any more than do erections evoked by somesthetic stimulation. The fact that NCE was no more impaired by RF lesions than by excitotoxic lesions in the MPOA indicates that neither intrinsic neurons nor fibers of passage through the MPOA are essential to the normal expression of NCE. It is possible, therefore, that the neural pathways mediating NCE do not originate in or pass through the MPOA. Our data, however, do not warrant the inference that the MPOA does not normally participate in regulating erection. The MPOA may lie on one of two or more parallel pathways through which erection may be effected by nonsomesthetic sexual stimulation.

\section{Males with MPOA lesions were sexually aroused}

The undiminished incidence of NCE in MPOAx males is the clearest evidence to date that such males are in fact sexually aroused by estrous females. Previous studies had suggested that MPOAx males are motivated to copulate (Szechtman et al., 1978; Hansen et al., 1982, 1984; Everitt and Stacey, 1987; Everitt, 1990). Still lacking, however, is a clear account of why MPOAx males exhibit their apparent copulatory apraxia. There is no basis for rejecting the hypothesis, proffered by Giantonio et al. (1970), that 


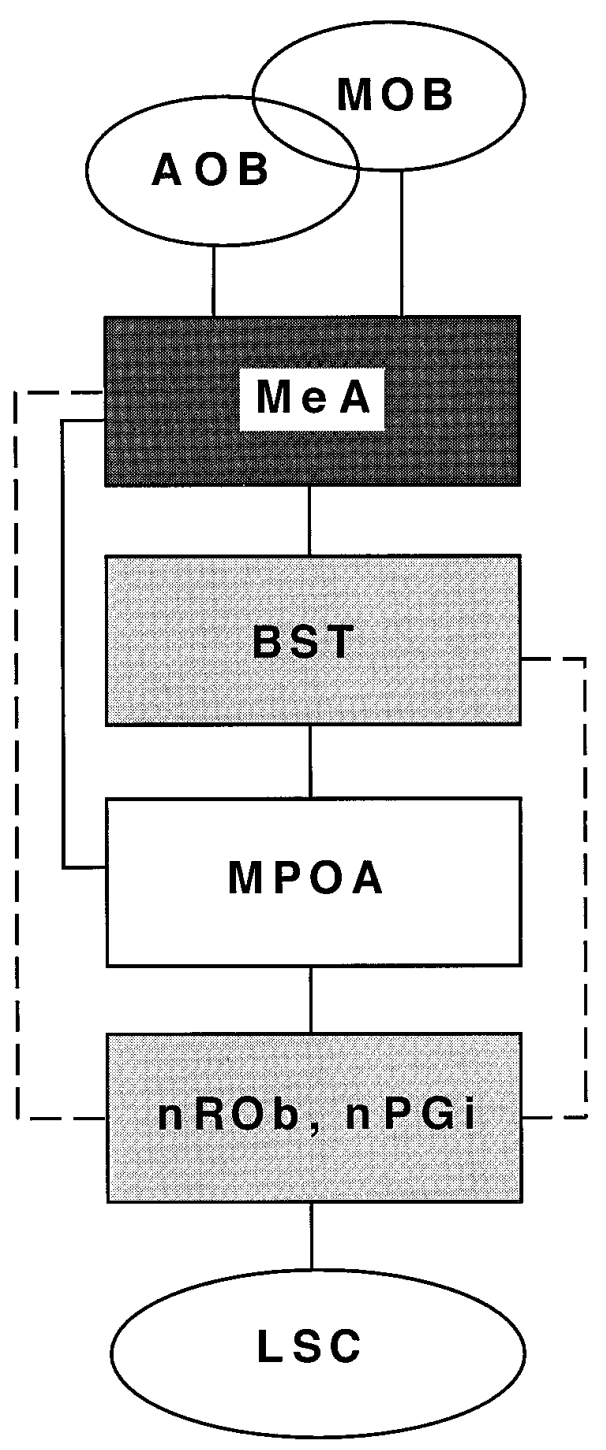

Figure 10. Major structures in the neural pathway from olfactory bulbs to spinal cord (adapted from Shipley et al., 1995), and estimates of their importance in the regulation of NCE. The roles of structures depicted as ovals remain to be explored. The roles of each of the rectangular structures have been explored in at least one experiment, and the rectangles are shaded in approximate proportion to their importance in the process of NCE. Lesions in MeA eliminate NCE (Kondo et al., 1997), lesions in BST impair NCE (Experiment 4), and lesions in MPOA have no reliable effect (Experiments 1-3). Preliminary reports on medullary structures suggest moderate facilitation by lesions in $n R O b$ (Kondo et al., 1996), but no reliable effect from $n P G i$ lesions (Liu et al., 1995). Solid lines indicate known direct projections, usually reciprocal, between structures. Dashed lines indicate hypothetical extra-MPOA projections, not necessarily direct ones, based primarily on the apparent redundancy of the $M P O A$ to the process of NCE. No attempt has been made to distinguish excitatory from inhibitory influences, although some of those from the medulla to $L S C$ are almost surely inhibitory. $A O B$, Accessory olfactory bulb; $B S T$, bed nucleus of stria terminalis; $L S C$, lumbosacral spinal cord; $M O B$, main olfactory bulb; $M e A$, medial amygdala; $M P O A$, medial preoptic area; $n P G i$, nucleus paragigantocellularis; $n R O b$, nucleus raphé obscurus.

the MPOA is essential for integrating afferent impulses and for organizing the sequential copulatory responses; however, both the incidence of NCE and its motor pattern appeared unaltered by MPOA lesions in rats (Experiments 1-3). Similarly, rhesus males with MPOA lesions continue to engage in normal levels of apparently well coordinated masturbation (Slimp et al., 1978). To- gether, these data suggest that the sexual disorder that results from MPOA lesions is specific to copulation, leaving intact the sensory integration, sequential motor patterning, and autonomic responding that is necessary for other sexual responses.

\section{BST plays an important role in mediating NCE and copulation}

BST lesions impaired the copulatory behavior of male rats (Experiment 4). The specific deficits, i.e., longer interintromission intervals and ejaculatory latencies, were generally consistent with previous studies (Giantonio et al., 1970; Emery and Sachs, 1976; Valcourt and Sachs, 1979; Claro et al., 1995). (We interpret the reduced percentage of MPOAx males achieving ejaculation as being secondary to changes in these measures in the face of time-limited tests.) There have been some reports (Emery and Sachs, 1976; Valcourt and Sachs, 1979; Claro et al., 1995) that BSTx males require more intromissions before ejaculating, but this effect was not evident in this study. Particularly relevant is that the BST and Sham groups had similar intromission ratios, an indication that erection adequate for vaginal insertion was maintained despite the lesions. These data imply that substantial lesions in the BST do not preclude normal erection in copula.

In contrast, males with BST lesions had a major deficit in the occurrence of erection in response to remote cues from estrous females. BSTx males had a much lower incidence of NCE, and when they did exhibit NCE, they had longer erection latencies than control males. The NCE deficits were generally larger in males with lesions affecting both the rostral and the caudal BST (BSTx-L) than in males with lesions limited to the rostral BST (BSTx-S), but this difference was not reliable in both tests.

It is not yet possible to do more than conjecture about why BST lesions impair NCE. The lesion and control groups were similar in the number of nose pokes displayed, suggesting that the deficit in NCE was not attributable to reduced attention to the female. The integrity of the MeA is essential for the expression of NCE (Kondo et al., 1997), and there are rich reciprocal connections between the MeA and the BST (Alheid and Heimer, 1988; Alheid et al., 1995). Both structures are important parts of the olfactory pathway (Kevetter and Winans, 1981a,b), and volatile odors from estrous females are the critical stimuli for evoking NCE (Sachs, 1996). Thus, lesions in MeA or BST may impair NCE by interfering with the central processing of olfactory cues. This cannot be the whole story, however, because one then might expect a progressive effect of lesions as one moves centrally and caudally from the sensory organs. That does not seem to be the case. Surgery intended to achieve total denervation of the main and accessory bulbs severely impaired NCE, but did not eliminate it (Edwards and Davis, 1997).

\section{An extra-MPOA pathway mediating NCE?}

Collectively, these and related experiments suggest that the neural mediation of NCE cannot be traced in a straightforward way along the traditional pathway from olfactory bulbs to amygdala to MPOA to spinal cord (Fig. 10). As already noted, lesions in the $\mathrm{MeA}$, which receives direct projections from the main and accessory bulbs, eliminated NCE (Kondo et al., 1997), whereas lesions in the BST, another step removed from the periphery, again had a more moderate effect (Experiment 4). Finally, the MPOA receives projections from $\mathrm{MeA}$ via the stria terminalis and the BST and, more directly, through the amygdalofugal pathway. Yet MPOA lesions do not disrupt NCE (Experiments 1-3). It follows logically that some MeA and BST neurons that are essential for NCE do not project to or through the MPOA, but rather exert their effects on NCE via an extra-MPOA pathway (Fig. 10). 


\section{REFERENCES}

Alheid GF, Heimer L (1988) New perspectives in basal forebrain organization of special relevance for neuropsychiatric disorders: the striatopallidal, amygdaloid, and corticopetal components of substantia innominata. Neuroscience 27:1-39.

Alheid GF, de Olmos JS, Beltramino CA (1995) Amygdala and extended amygdala. In: The rat nervous system, second edition (Paxinos G, ed), pp 495-578. San Diego: Academic.

Blackburn JR, Pfaus JG, Phillips AG (1992) Dopamine functions in appetitive and defensive behaviors. Prog Neurobiol 39:247-279.

Canteras NS, Simerly RB, Swanson LW (1995) Organization of projections from the medial nucleus of the amygdala: a PHAL study in the rat J Comp Neurol 360:213-245.

Claro F, Segovia S, Guilamón A, del Abril A (1995) Lesions in the medial posterior region of the BST impair sexual behavior in sexually experienced and inexperienced male rats. Brain Res Bull 36:1-10.

Courtois FJ, Macdougall JC (1988) Higher CNS control of penile responses in rats: the effect of hypothalamic stimulation. Physiol Behav 44:165-171.

de Groat WC, Booth AM (1993) Neural control of penile erection. In: The autonomic nervous system, $\mathrm{Vol} 3$, Nervous control of the urogenital system (Maggi CA, ed), pp 467-524. London: Harwood Academic.

Edwards DA, Davis AB (1997) Deafferentation of the olfactory bulbs of male rats reduces erection to remote cues from females. Physiol Behav, in press.

Emery DE, Sachs BD (1976) Copulatory behavior in male rats with lesions in the bed nucleus of the stria terminalis. Physiol Behav 17:803-806.

Everitt BJ (1990) Sexual motivation: a neural and behavioral analysis of the mechanisms underlying appetitive and copulatory responses of male rats. Neurosci Biobehav Rev 14:217-232.

Everitt BJ, Stacey P (1987) Studies of instrumental behavior with sexual reinforcement in male rats (Rattus norvegicus). II. Effects of preoptic area lesions, castration, and testosterone. J Comp Psychol 101:407-419.

Giantonio GW, Lund NL, Gerall AA (1970) Effect of diencephalic and rhinencephalic lesions on the male rat's sexual behavior. J Comp Physiol Psychol 73:38-46.

Giuliano F, Rampin O, Brown K, Courtois F, Benoit G (1996) Stimulation of the medial preoptic area of the hypothalamus in the rat elicits increases in intracavernous pressure. Neurosci Lett 209:1-4.

Hansen S, Drake af Hagelsrum LJK (1984) Emergence of displacement activities in the male rat following thwarting of sexual behavior. Behav Neurosci 98:868-883.

Hansen S, Köhler C, Goldstein M, Steinbusch HVM (1982) Effects of ibotenic acid-induced neuronal degeneration in the medial preoptic area and the lateral hypothalamic area on sexual behavior in the rat. Brain Res 239:213-232.

Hart BL (1968) Sexual reflexes and mating behavior in the male rat J Comp Physiol Psychol 65:453-460.

Heimer L, Larsson K (1966/1967) Impairment of mating behavior in male rats following lesions in the preoptic-anterior hypothalamic continuum. Brain Res 3:248-263.

Holmes GM, Holmes DG, Sachs BD (1988) An IBM-PC based data collection system for recording rodent sexual behavior and for general event recording. Physiol Behav 44:825-828.

Hull EM (1995) Dopaminergic influences on male rat sexual behavior In: Neurobiological effects of sex steroid hormones (Micevych PE, Hammer RP, eds), pp 234-253. Cambridge: Cambridge UP.

Hull EM, Du J, Lorrain DS, Matuszewich L (1995) Extracellular dopamine in the medial preoptic area: implications for sexual motivation and hormonal control of copulation. J Neurosci 15:7465-7471.

Kevetter, GA, Winans SS (1981a) Connections of the corticomedial amygdala in the golden hamster. I. Effects of the "vomeronasal amygdala.” J Comp Neurol 197:81-98.

Kevetter, GA, Winans SS (1981b) Connections of the corticomedial amygdala in the golden hamster. II. Effects of the "olfactory amygdala." J Comp Neurol 197:99-111.

Kondo Y, Yamanouchi K, Sakuma Y (1996) Effects of the raphe obscurus lesion in the medulla on penile erection and copulatory behavior in the male rats. 28th Annual Conference on Reproductive Behavior, Montreal, Canada, June 15-18.

Kondo Y, Sachs BD, Sakuma Y (1997) Importance of the medial amygdala in rat penile erection evoked by remote stimuli from estrous females. Behav Brain Res, in press.
Krane RJ, Goldstein I, Saenz de Tejada I (1989) Impotence. New Engl J Med 321:1648-1659.

Liu Y-C, Salamone JD, Sachs BD (1995) Differential role of three brain regions (MPOA, $\mathrm{PVH}$, and $\mathrm{NPGi}$ ) in rat erectile function. Soc Neurosci Abstr 21:1464.

Malsbury CW (1971) Facilitation of male rat copulation behavior by electrical stimulation of the medial preoptic area. Physiol Behav 7:979-805.

Mas M (1995) Neurobiological correlates of masculine sexual behavior. Neurosci Biobehav Rev 19:261-277.

Meisel RL, Sachs BD (1994) The physiology of male sexual behavior. In: The physiology of reproduction, 2nd edition, Vol 2 (Knobil E, Neill JD, eds), pp 3-105. New York: Raven.

Oomura Y, Yoshimatsu H, Aou S (1983) Medial preoptic and hypothalamic neuronal activity during sexual behavior of the male monkey. Brain Res 266:340-343.

Paredes R, Haller AE, Manero MC, Alvarado R, Ågmo A (1990) Medial preoptic area kindling induces sexual behavior in sexually inactive male rats. Brain Res 515:20-26.

Paxinos G, Watson C (1986) The rat brain in stereotaxic coordinates. San Diego: Academic.

Pellegrino LJ, Pellegrino AS, Cushman AJ (1979) A stereotaxic atlas of the rat brain. New York: Plenum.

Pfaus JG, Phillips AG (1991) Role of dopamine in anticipatory and consummatory aspects of sexual behavior in the male rat. Behav Neurosci 105:727-743.

Rosen RC, Beck JG (1988) Patterns of sexual arousal. New York: Guilford.

Sachs BD (1995a) Placing erection in context: the reflexogenic-psychogenic dichotomy reconsidered. Neurosci Biobehav Rev 19:211-224.

Sachs BD (1995b) Context-sensitive variation in the regulation of erection. In: The pharmacology of sexual function and dysfunction (Bancroft J, ed), pp 97-108. Amsterdam: Elsevier.

Sachs BD (1996) Volatile odors from inaccessible estrous females are necessary and sufficient to activate a species-typical sexual response in male rats. Soc Neurosci Abstr 22:1823.

Sachs BD, Garinello LD (1978) Interaction between penile reflexes and copulation in male rats. J Comp Physiol Psychol 92:759-767.

Sachs BD, Akasofu K, Citron JH, Daniels SB, Natoli JH (1994) Noncontact stimulation from estrous females evokes penile erection in rats. Physiol Behav 55:1073-1079.

Scalia R, Winans SS (1975) The differential projections of the olfactory bulb and accessory olfactory bulb in mammals. J Comp Neurol 161:31-56.

Shimura T, Yamamoto T, Shimokochi M (1994) The medial preoptic area is involved in both sexual arousal and performance in male rats: re-evaluation of neuron activity in freely moving animals. Brain Res 640:215-222.

Shipley MT, McLean JH, Ennis M (1995) Olfactory system. In: The rat nervous system, 2nd edition, (Paxinos, G, ed), pp 899-926. San Diego: Academic.

Slimp JC, Hart BL, Goy RW (1978) Heterosexual, autosexual and social behavior of adult male rhesus monkeys with medial preoptic-anterior hypothalamic lesions. Brain Res 142:105-122.

Stefanick ML, Davidson JM (1987) Genital responses in noncopulators and rats with lesions in the medial preoptic area or midthoracic spinal cord. Physiol Behav 41:439-444.

Szechtman H, Caggiula AR, Wulkan D (1978) Preoptic knife cuts and sexual behavior in male rats. Brain Res 150:569-591.

Valcourt RJ, Sachs BD (1979) Penile reflexes and copulatroy behavior in male rats following lesions in the bed nucleus of the stria terminalis. Brain Res Bull 4:131-133.

van de Poll NE, van Dis H (1979) The effect of medial preoptic-anterior hypothalamic lesion on bisexual behavior of the male rats. Brain Res Bull 4:505-511.

Weller KL, Smith DA (1982) Afferent connections to the bed nucleus of the stria terminalis. Brain Res 232:255-270.

Winn P (1991) Excitotoxins as tools for producing brain lesions. In: Lesions and transplantation (Conn PM, ed), pp 16-27. San Diego: Academic.

Yahr P, Gregory JE (1993) The medial and lateral cell groups of the sexually dimorphic area of the gerbil hypothalamus are essential for male sex behavior and act via separate pathways. Brain Res 631:287-296.

Zuckerman M (1971) Physiological measures of sexual arousal in the human. Psychol Bull 75:297-329. 